\title{
Separating, replacing, intersecting: The influence of context on the construction of the medical-nursing boundary
}

\author{
Dr Elisa G. Liberati
}

Cambridge Centre for Health Services Research (CCHSR), Department of Public Health and Primary Care, Forvie Site, Robinson Way, Cambridge CB2 OSR, UK.

E-mail: egl24@medschl.cam.ac.uk

\begin{abstract}
The distribution of work, knowledge, and responsibilities between doctors and nurses is a longstanding object of interest for medical sociologists. Whereas the strategies through which nurses and doctors construct their professional boundary have been thoroughly examined, little is known about why the regulation of the medical-nursing boundary varies across care settings. In the article, I argue that this gap in knowledge can be attributed to insufficient examination of the 'negotiation context' (Strauss, 1978), namely the features of the social and organisational environment that directly affect doctor-nurse boundary negotiations. Adopting a negotiated order perspective, and drawing data from a hospital ethnography, the article describes the different ways of constructing the medical-nursing boundary (separating, replacing, and intersecting) which were observed in three different care settings (a neurology ward, a neurosurgical ward, and an intensive care unit). Constant comparison of the observed interactional patterns led to the identification of three factors that significantly affected the construction of the medical-nursing boundary, specifically: patients' state of awareness, the type of clinical approach adopted by nurses and doctors, and the level of acuity on the ward. The article advances our knowledge of the medicalnursing boundary by shedding light on its flexible and contextual nature and by adding further nuance to the boundary-blurring/boundary-reinforcing dichotomy. New features of the 'negotiation context' are identified that enable more convincing explanations of why the medical-nursing boundary varies across care settings. Finally, the study advances the negotiated order theory by offering a framework for considering the structural differences that shape local negotiations.
\end{abstract}

Key words: Italy; professional boundaries; interprofessional working; medical-nursing boundary; ethnography. 


\section{Introduction}

During the last four decades changes in health systems have significantly challenged medical dominance in relation to nursing (Tousijn, 2000; Nancarrow and Borthwick, 2005). The shortage of doctors and the rising of healthcare demand has led to increased 'task shifting' (WHO, 2007). In some high-income countries the role of nurses has been extended to include traditionally medical tasks, such as the prescription of routine medication. Furthermore, substantial reforms in nursing education and policies, together with the rise of new nursing ideologies (Beardshaw and Robinson, 1990), have allocated a more prominent and autonomous role to nurses (Nancarrow and Borthwick, 2005). These changes created potential tensions in the medical-nursing interface, sometimes sparking jurisdictional battles (Abbott, 1988).

Mirroring this increased complexity, social scientists have gradually moved away from the overdeterministic model of the 'medical dominance' (Freidson, 1970) to adopt theoretical perspectives that enable a more nuanced understanding of the interplay between nursing and medicine. Negotiated order is one such perspective (Strauss, 1978; Strauss et al., 1997). Studies informed by negotiated order have demonstrated that nurses influence patient care in ways that contradict their place in formal organisational hierarchies and participate in medical work tacitly and flexibly (Hughes, 1988; Allen, 1997; Nugus et al., 2010). Emphasising the "delicate ordering of healthcare work" (Hindmarsh and Pilnick, 2002:141), negotiated order concepts have had tremendous value for the sociology of medical professions, enabling images of nursing and medicine that are more congruent with the reality of current clinical work.

This study is grounded in this theoretical legacy. It utilises the notion of 'negotiation context' (Strauss, 1978) to develop a theory of why the construction of the medical-nursing interface varies across care settings and in response to which contextual factors - a remarkably under-investigated question in the medical sociology literature.

\section{Negotiated order and the medical-nursing boundary}

At the centre of the negotiated order approach is the argument that daily work is constructed in ways that only partly derive from regulatory frameworks and formal structures, and are also the product of enduring interactions of all actors involved in the exercise of agency and the concurrent creation of a relatively stable social order (Strauss, 1978; Fine, 1984; Nugus et al., 2010). Although structures and rules shape organisational life, these are not unescapably prescriptive nor peremptorily constraining; in many instances rules are cited selectively, stretched, or even ignored by individuals at convenient moments (Hughes, 1984; Strauss, 1978).

This approach leads to a conception of division of labour as a process of social interaction upheld by its participants through their daily work (Strauss et al., 1997). Jurisdictions are not self-evident and need to be actively negotiated and claimed within a system of work (Abbott, 1988; Allen, 1997). Individuals play an active, though not necessarily decisive, role in the making, negotiating, and maintaining of professional boundaries - what has been defined 'boundary-work' (Abbott, 1988). Accordingly, professional boundaries and identities are tightly intertwined. Since individuals derive their identities and self-esteem from their professional work, they seek to maintain control over the 
areas of work that affords them symbolic rewards, while discarding 'dirty work' (Hughes, 1984; Kellogg, 2014).

When applied to the study of the medical-nursing boundary the negotiated order approach has demonstrated that the real-life work of nurses cannot be reduced to their formal job descriptions or organisational role (Dingwall and Allen, 2001). Hughes' (1988) study of an accident and emergency unit showed that nursing work in the processes of patient categorisation moved close to the medical task of diagnosis. Similarly, Tjora (2000) determined that in order to manage requests for medical assistance when doctors were unavailable, the nurses of a communication centres drew on their professional experience and assembled knowledge from different clinicians to diagnose patients' conditions over the phone. The medical-nursing boundary can be constructed through either explicit negotiations (namely vis-à-vis disputes concerning professional boundaries) or de facto boundary blurring, which refers to nurses informally taking over doctors' work to respond to workplace pressures (Allen, 1997:551). In sum, negotiated order studies show that nurses may participate in medical work tacitly and flexibly, and the boundaries between curing and caring may be more blurred than is formally acknowledged (Baumann et al., 1998; Chambliss, 1996; Salhani and Coulter, 2009).

Negotiated order studies also challenge the static conception of power implied by the 'medical dominance' model (Freidson, 1970), demonstrating that nurses may exercise power in ways that exceed passive influence attempts. Various scholars suggested that the medicine-nursing power relationship may not be a 'zero-sum' one; forms of collaborative power between nurses and doctors may exist (Nugus et al., 2010) and strategies may be developed to increase the influence of both groups in the wider network of healthcare practitioners (Carmel, 2006).

The nuanced description of medical and nursing work offered by this body of literature has tremendous value, demonstrating how close examination of daily workplace interactions is key to grasping the flexible patterns of activities underpinning the medical-nursing boundary. Yet, since the focus of negotiated order studies has traditionally been on how the medical-nursing boundary is regulated in individual care settings, little explanation is offered of why these strategies vary across contexts. As a result, when taking stock of this body of research, some contrasting findings become apparent.

In her study of a medical and a surgical hospital ward, Allen (1997) observed that the fragmented presence of doctors frustrated nurses' attempts to sustain a rigid division of labour, facilitating de facto boundary blurring. Thus, the author suggests, nurses were more likely to engage in informal (de facto) boundary blurring when doctors were unavailable, while acting within their formal role when doctors were physically present. But Carmel's study of an intensive care unit portrays doctors and nurses purposively blurring their professional boundary when doctors were physically present. Carmel framed this as a strategy to increase the influence of both groups in relation to other specialties and the wider hospital context (Carmel, 2006). Nugus et al. (2010) found that the acuity of the care setting was a mediating variable in balancing collaboration amongst healthcare professionals, with acute care settings characterised by a greater degree of medical dominance than non-acute settings. Yet Hughes's (1988) aforementioned study suggested the opposite pattern.

I argue that these apparently divergent findings can be attributed to insufficient examination and theorisation of the contextual factors which directly impact the regulation of the medical-nursing 
boundary. The negotiated order approach, and particularly the notion of 'negotiation context' (Strauss, 1978), offers a promising framework for this analysis.

Although emphasising the 'primacy of work' in shaping the division of labour that forms around it (i.e. the idea that it is daily work practices themselves that shape division of labour [Strauss et al., 1997]), advocates of the negotiated order approach do recognise the constraining role played by the context in which interactions occur. Strauss himself emphasised the existence of structural properties which directly influence the course of workplace negotiations, such as the number of negotiators, who they represent, the sequence and frequency of negotiations, and the issues at stake in the negotiations. The author named these structural properties 'negotiation context' (1978). Busch (1982) added further specification to this argument. He introduced the concept of sedimentation to explain the process whereby the outcome of previous interactions becomes part of the structural context and acquires a non-negotiable and taken-for-granted status. Nevertheless, these concepts have rarely been mobilised to explain variations in the medical-nursing boundary. If this continues, studies in this field risk being criticised for being unable to deal with the limiting factors in negotiation settings, a criticism frequently made of negotiated order theory more broadly.

This is where the present study seeks to contribute. Drawing data from a hospital ethnography, it aims to identify the social, contextual, and organisational factors that directly affect the negotiation of medical-nursing boundary (i.e. the properties of the 'negotiation context') and examine how they operate in different care settings. This will be accomplished by comparing the processes through which the medical-nursing boundary is constructed in different hospital wards.

\section{Research design}

\subsection{Context}

Data for this study are drawn from a broader ethnographic research conducted in a multidisciplinary public hospital in Italy, between February 2014 and April 2015. The original aim of the research was to examine how inter-professional and inter-disciplinary boundaries in medicine and nursing were negotiated in the context of a policy-driven organisational change (Reference-omitted-to-preserveanonymity). For the current purposes, I will focus mainly on the data that contribute to a more nuanced understanding of the 'negotiation context' and its role in shaping the nursing-medical boundary.

Two well documented features of the Italian healthcare context make Italy an analytically interesting case for the purposes of this study, namely, the relative lack of standardisation of nursing practice (Yamaguchi, 2004; Goopy, 2005) and the mismatch between legal structures and the daily experiences of the nurses (Barazzetti et al., 2005; Sansoni, 1995; Tousijn, 2000). Under these circumstances it seems likely that variations across care settings in how nursing jurisdiction is accomplished would be particularly pronounced, thus giving sharp profile to the salient properties of the 'negotiation context' (see Box 1 for further details). 


\subsection{Setting and participants}

Data were collected in a neurology ward, a neurosurgical ward, and an intensive care unit (ICU); participants included 10 surgeons, 8 neurologists, 10 intensivists, and 45 nurses with different disciplinary backgrounds. The above care settings offered a promising opportunity to address my research question, for two main reasons. First, the three wards were characterised by different degrees of physical attendance by doctors and nurses and by different levels of acuity and patient turnover, two contextual features that have been interpreted in contrasting ways by existing studies. Second, the sample included professionals with different disciplinary backgrounds, who were likely to be characterised by different professional identities and daily working patterns, thus presumably affecting the negotiation context (Kellogg, 2014).

\subsection{Data collection}

I adopted an ethnographic approach to generate in-depth and context-sensitive knowledge and to develop an understanding of the practical accomplishment of the medical-nursing boundary (Hammersley and Atkinson, 2007). Data collection activities included observations of routine care activities in the aforementioned wards (Table 1), participation in medical/nursing briefing and handovers, and frequent conversations between the researcher and nurses and doctors. Conversations took place on the wards, in nursing stations, doctors' offices, or ward kitchens; for their prolonged duration and depth of reflections achieved, informal conversations often resembled a formal interview. Observations and conversations aimed to capture: (1) how doctors and nurses organised their activities in relation to others (e.g., accepting or delegating tasks); (2) how doctors and nurses described and interpreted their jurisdiction in relation to their professional identity; (3) whether overt boundary negotiations or informal boundary blurring occurred. Informed consent was obtained from participants, who were informed about my background as a social scientist. The study received ethical approval.

[Table 1]

\subsection{Data analysis}

Ethnographic field notes were transcribed at the end of every fieldwork day and organised into three sections: observational notes (detailed description of interactions and other events), methodological notes (reflections regarding the techniques of data collection), and theoretical notes (provisional theoretical explanation to the observed events) (Gobo, 2008). Data gathering and analysis proceeded concurrently, meaning that some of the most important insights emerged within the fieldwork (in the form of theoretical notes), and were then re-examined and linked to the entire corpus of data. Participants' feedback increased face validity and added relevant insights to the corpus of data.

Data analysis was informed by the constant-comparative method (Charmaz, 2014). The data for each care setting were carefully examined to ensure that they were each understood in terms of their own context. I then compared data across contexts in order to develop higher-level concepts and to 
identify reasons for their differences and similarities. Although mainly inductive, this analysis was not purely data-driven. Concepts derived from the negotiated order theory and the sociology of professions (e.g. negotiations, social order, professional boundaries, professional identities) were introduced in the analysis to enable the construction of a descriptive and explanatory framework of the medical-nursing boundary.

The descriptive component (Section 4.1) illustrates three different modalities of constructing the medical-nursing boundary; it articulates the boundary-blurring/boundary-reinforcing strategies adopted within each and provides examples of the activities that underpinned the negotiation of the medical-nursing interface. The explanatory component (Section 4.2) focuses on the role of the 'negotiation context'; it examines the structural properties that shaped doctor-nurse boundary negotiations in each care setting, thus explaining the differences between the three. Data are presented in the form of 'thick descriptions' (Gobo, 2008) and enriched with extracts from my field notes. In translating these from Italian to English, I engaged with native English speakers to ensure accuracy and to recreate meaningful linguistic nuances.

\section{Findings}

\subsection{Separating, replacing, intersecting: The situated nature of the nursing-medical boundary} Observations of routine clinical practice in the three selected care settings lead to the identification of three different interactional patters between doctors and nurses, namely, different ways in which the medical-nursing boundary was negotiated and constructed. I named these patterns separating, replacing, and intersecting. Each pattern was observed mainly in one care setting (the neurology ward, neurosurgery ward, and ICU, respectively).

\section{- Separating}

The separating pattern was prevalent in the neurology ward and mainly characterised the interactions between neurology nurses and neurologists. Here, both nurses and doctors spent the majority of their work shift in the neurology ward. Despite the communal working space, the medical and nursing jurisdictions were clearly separated, giving rise to a sharp division between the realms of curing and caring. Doctors expected nurses to dedicate their efforts only to assistant tasks (e.g. administrating medications, toileting, controlling patients' eating, conversing with patients) and to refrain from contributing to the diagnostic process. The only information that doctors directly asked of nurses concerned these assistant tasks; transforming such information into data relevant to patients' treatment was agreed to be the doctors' job. Accordingly, neurology nurses usually avoided taking action or asking questions with respect to patients' treatment and medication. These findings echoed those of Allen (1997), who observed nurses acting within the boundary of their formal role when doctors were physically present.

[Observation in neurology ward]

Two neurologists ( $V$ and $F$ ) and one nurse (E) are doing the rounds visit. They stop in front of the room of a newly admitted patient. $V$ says to $F$, "[The patient] has a continuous infusion". $V$ turns to nurse E and asks, "Did he arrive here like this?"; E nods her head. $V$ then turns again to $F$ and proposes to start treatment with [medication name]. The neurologists go on discussing the treatment, while $E$ listens in silence. Then, $F$ asks of $E$ how the blood pressure is. $E$ replies 
that the pressure is still high, but better than this morning. $V$ asks $E$ if the patient is refusing to eat, and the nurse confirms. (...) The neurologists enter the patient's room and start to visit him. E enters too and stops in one corner of the room, taking notes while listening to the visit.

In their workplace conversations, neurology nurses hardly ever mentioned or discussed treatment choices, and they attributed high value to their assistant role and 'emotion work' (Dingwall and Allen, 2001; Hochschild, 2003). As the following extract shows, neurology nurses were very careful not to encroach on medical terrain even when not interacting with doctors.

[Observation in the neurology ward. Nurse $L$ has worked for 5 years in a surgical ward and, since two months, she has been training in the neurology ward]

In the ward corridor, Nurse $L$ encounters Nurse $B$ (a senior neurology nurse) and shows her a patient's medical notes.

Nurse L: "An enema at this time of the day? It looks weird. It may be a mistake...?"

Nurse B: "If it's written there it must be for a reason. I don't know this patient but I think you should follow what's written there"

Nurse L: "Are you sure? It doesn't make much sense to me..."

Nurse B: "If you suspect there's a mistake, you should go ask doctor F (neurologist), he prescribed it."

The separating pattern reflected a high degree of medical dominance (Freidson, 1970): the autonomy of neurology nurses was very limited in practice and the medical-nursing boundary was clear and impermeable.

- Replacing

The replacing pattern was prevalent in the surgical ward and mainly characterised the interactions between surgical nurses and neurosurgeons. Here, doctor-nurse interactions were less frequent than in the neurology ward. Being in the operating rooms for most of their work shift, surgeons spent very little time with in-ward patients, and nurses were the only care providers constantly present in the neurosurgery ward. Some surgical nurses routinely took over surgeons' tasks, such as prescribing basic drugs, deciding when to contact other specialists for a consultation, removing stiches, and handling surgical wounds. During the daily ward-rounds, surgeons relied completely on the nurses to acquire the necessary information on patients.

[Observation in surgical ward]

Surgeon S arrives in the surgical ward. As usual, he recruits a nurse (F) and they start the wardround. $S$ is visibly in a hurry. $S$ and $F$ enter the first patient's room, $S$ says hi to the patient and then turns to nurse F: "So, what do we have here?"

$F$ takes his nursing record and updates $S$ on the patients' conditions, the specialists' visits he received, the state of the surgical wound, the symptoms he showed in the last few hours. Once F has finished, the surgeon turns to the patient and asks: "So everything's OK then..?" (...)

Once in the corridor, $S$ asks $F$ if this patient is going to need physical rehabilitation.

F: "Your colleague has written that rehabilitation is not needed, but I think we should reconsider... The gait is very unsteady..."

S: "If it's already written down I can't do anything"

F: "Can we at least ask for the physiotherapist opinion?" 
$S$ hesitates, but then he agrees with the nurse and completes the form for the consulting request.

All the surgeons expected the nurses to replace them in the aforementioned activities. While informal boundary blurring occurred mostly when doctors were absent from the ward (thus confirming Allen's [1997] findings), some senior nurses openly questioned surgeons' decisions even when the surgeons were present. In such cases, as shown by the previous extract, surgeons often took nurses' concerns into consideration.

In the surgical ward, nurses were provided with access to tasks that fell outside their formal jurisdiction, but that surgeons expected them to perform nonetheless. The social order of neurosurgery shaped nursing role in such a way that nurses routinely blurred their professional boundary to replace surgeons and undertake some medical tasks.

Several surgical nurses I conversed with felt rewarded by this informal expanded role and perceived it as consistent with their professional identity as expert and autonomous nurses. Fewer nurses regarded this distribution of work and responsibilities as illegitimate.

[Conversation with surgical nurses]

Researcher: "I noticed that you suggested to (surgeon) which drugs to prescribe for (patient name)"

Senior nurse: "Yes... This happens a lot here. Surgeons have a hard job to do themselves in the operating room, so we have to handle the ward ourselves. They rely on us. Do not take this to the letter but a doctor once told me that it's thanks to us that no patient has died in this ward so far! (...) It's nice because we grow professionally, we become more autonomous. But the patient is completely under our responsibility."

Staff nurse: "I really don't agree with this... We are not being paid to do this. And most surgeons don't even check on what we do. Sometimes I'm really tempted, you know? I'd like to try them, to say something that really doesn't make sense... Just to see if they realise it or not."

Yet, notably, even these frustrated nurses took over many surgeons' tasks in their daily job, explaining that this was the only way to guarantee patients' circulation in the hospital (Allen, 2014). In the replacing pattern, boundary blurring was often explained as being "for the sake of the patients".

- Intersecting

Finally, the intersecting pattern was prevalent in the ICU. In this case, though both doctors and nurses were constantly present on the ward, the medical-nursing boundary in the ICU was blurred and permeable (thus diverging from Allen's [1997] findings). By reading medical reports and participating in doctors' conversations, ICU nurses were able to autonomously establish their duties, and they often anticipated doctors' requests. Moreover, ICU nurses sometimes corrected doctors' choices by providing new information about the patients. Some nurses (mostly, but not only, senior ones) actively participated in the diagnosis, watching and commenting on medical reports (e.g. CT scans, $\mathrm{x}$-rays) together with the intensivists.

[Observation in ICU] 
Nurse D opens the CT scan in the computer and shows it to intensivist $M$.

Nurse: "Look, look where it goes out from! I think it's a glioblastoma... look at how it lifted the operculum!"

Intensivist: "Do you think so? But that doesn't explain the seizure."

Nurse: "Ok M, but ... [she opens another CT scan] ...look at the previous picture... it really moved the operculum!"

Intensivists granted ICU nurses with access to knowledge that became crucial in nurses' daily work. In light of their shared knowledge and experiences, intensivists accepted nurses' corrections and indications, and regarded them as fundamental to avoiding errors rather than a challenge to their authority. Intensivists freely acknowledged the skills and competence of nursing staff and their crucial role in the ICU. Moreover, boundary blurring, in this case, was reciprocal: nurses and intensivists substituted for each other in an array of tasks and frequently engaged in reciprocal double-checking of their actions. Both doctors and nurses made an effort to attend one another's handovers, suggesting that the kind of information exchanged by the two professional groups was regarded as similarly helpful to both. Intensivists and nurses often referred to each other using the possessive adjective ("my doctors", "my nurses") in a way that emphasised their mutual level of trust and sensitivity to one another's viewpoints. Senior staff confirmed that this relationship existed even before the changes in formal legislation (Box 1). These observations resonate with Carmel's (2006) conclusion that some specific features of the ICU (i.e. the contrast between the ICU and the general wards, an informal working environment, and close working interactions) may blur the medical-nursing boundary.

[Conversation with intensivist]

Researcher: "I saw that nurse A warned you about something strange in the prescription for the patient"

Intensivist: "Yes, it wasn't an error in this case, but this kind of reciprocal feedback is absolutely crucial. We need to listen carefully to what nurses say; sometimes they notice in advance that something bad is going to happen... It's also thanks to this double-checking that we are able to solve it before it's too late."

ICU nurses seemed to consider this interpretation of their role as legitimate; even if the medicalnursing boundary was blurred, nurses did not think that doctors were "dumping" their duties on them. Boundary blurring in the ICU did not lead to nurses replacing doctors in their tasks (an in the neurosurgical ward) but rather created areas of overlap and intersection, a condition that allowed a closer collaboration with doctors and strengthened solidarity and alliance.

4.2 Why did the medical-nursing boundary vary across care setting? Disentangling the role of the 'negotiation context'

I argue that the different ways of constructing the medical-nursing boundary in neurology, neurosurgery, and the ICU were associated with the presence of different 'negotiation contexts' (Strauss, 1978). Three factors in particular appeared to influence doctor-nurse interactional patterns: the level of acuity of the ward, patients' state of awareness, and the type of clinical approach (holistic versus specialised) adopted by nurses and doctors. 
In acute care contexts, with a rapid patient turnover, nurses and doctors justified their boundary blurring by mentioning the severity of patients' conditions, their clinical instability, and the potential for emergencies or unexpected events. This was the case for both the units where doctors were constantly present (ICU) and for those where doctors' presence was more fragmented (neurosurgery ward); the speed of events and the required timeliness of actions made it virtually impossible for nurses and doctors to maintain clearly separated jurisdictions.

[Conversation with nurse $D$, surgical ward, and nurse $F, I C U$ ]

Nurse D: "If the patient has a seizure, you have to do something! You cannot just wait for someone to take over and tell you what to do..."

Nurse F: "Same for us. Everything goes so fast... The more you spend time in the ICU, the more you learn that anticipating is crucial. Our patients need us to do that..."

Emergencies were much less frequent in the neurology ward and, when they occurred, nurses could count on the constant presence of the doctors to manage the situation. Therefore, in the neurology ward, nurses' work was more predictable, routine-oriented, and adherent to nurses' formal role; boundary blurring was uncommon. Nurses' daily activities differed sharply across the three care settings depending on their respective level of acuity. This effected the extent to which engaging in tasks that fell under doctors' jurisdictions was regarded by the nurses as an illegitimate encroachment (i.e. in the neurology ward) or as a constitutive aspect of their professional identity (i.e. in the surgical ward and ICU). These observations resonated with those of Hughes (1988) and Allen (1997), who suggested that de facto boundary blurring can be amplified by the urgency of treatments. By comparing urgent and non-urgent care settings, this study provides further proof of the impact of this contextual feature on the regulation of medical-nursing boundary.

\section{- Patients'state of awareness}

Strauss et al. (1987) remind us that patients participate in the division of labour as much as professionals do. Although widely accepted, this argument has rarely been mobilised to explain the construction of the medical-nursing boundary. My observations revealed that patients' behaviour and state of awareness affected the regulation of the medical-nursing boundary in important ways.

Where the medical-nursing boundary was clear and impermeable (as in the neurology ward), patients were clinically stable and presented a higher state of awareness than in the other two care setting; therefore, patients interacted more often with both doctors and nurses. When speaking to the nurses, patients revealed their fears, wishes, and personal needs, and asked questions that they hardly ever directed to doctors. Thus nurses were provided with access to a significant body of 'social knowledge' regarding patients (Hughes, 1988) and with opportunities to engage in 'emotion work' (Hochschild, 2003), and these aspects became central to their professional identity. In contrast, these intimate conversations hardly ever occurred with doctors, whom patients generally asked about clinical information related to their care plan. Ultimately, in the neurology ward, the very fact that patients were awake and communicated with nurses and doctors in such different ways reinforced the medical-nursing boundary by further segregating the realms of curing and caring. 
[Observation in neurology ward]

Nurse $T$ enters the meeting room where some neurologists are filling in their medical records. $T$ turns to neurologist $H$ and says: "[patient's name] has been crying all morning. She says that she's been having diarrhoea for two months, she's very worried..."

$H$ interrupts: "Diarrhoea does not seem to me like a neurological problem"

Nurse: "Well, her family has a history of bowel cancer; she'd like to speak with a doctor to have some clarifications about the medications she's taking, but she's afraid to ask."

$\mathrm{H}$ : "Tell her that she has nothing to worry about, there's no correlation between the two things."

In the ICU and the surgical ward, the situation was different; patients were mainly unconscious or poorly respondent, and once they started to recover their interactional abilities patients were moved to other wards or discharged from the hospital. Patients acting as 'unrespondent ill bodies' provided nurses with fewer opportunities to take on a purely caring role; instead, nurses' main internalised responsibility was to provide timely clinical intervention. Thus, in the ICU and the surgical ward patients' low state of awareness contributed to blurring the medical-nursing (curingcaring) boundary.

Notably, some nurses appeared at ease with this role; the perceived similarity with doctors' roles seemed to reinforce their expertise and power, while 'emotion work' was belittled as work that could be performed by unqualified staff. As found by Nyström (2002), 'emotion work' failed to strengthen the self-esteem and professional identity of these nurses to the same degree that medical tasks did. In the following extract, "I" is the researcher.

[Conversation in the ICU, ward kitchen]

Nurse $G$ is speaking about his wife, who works a nurse in the neurology ward. I ask $G$ if he would like to work there too.

Nurse G: "Oh, please no! So boring..."

I: "Why boring?"

Nurse G: "Nothing happens there. You have to do only what the doctor writes, prescriptions are always perfect, and patients stay in the ward for a lot of time... Nurses are this close to getting these patients a manicure! I like the hands-on work more, I feel like I'm actually doing something here, you know?"

\section{- Holistic versus specialised clinical approaches}

Historically, medicine has been associated with a disease-focused clinical approach that relies on specialised knowledge, whereas nursing is seen as a holistic and patient-centred profession (Foley and Faircloth, 2003). Although this dualism does not necessarily do justice to the complexity of nursing practice (Baumann et al., 1998; Allen, 2014), health professionals often rely on it to legitimise their professional identity and to emphasise differences with others (Foley and Faircloth, 2003). In the present study many nurses purposely used the contrast between specialised and holistic clinical approach to reinforce the boundary between themselves and neurologists - seen as highly 'specialised' doctors - and to position themselves as closer to intensivists. Nurses suggested that neurologists applied a narrow clinical approach, focusing on specific symptoms and leaving to the nurses the duty of contextualising medical decisions within patients' broader care process.

[Conversation with nurse $D$, surgical ward] 
Problem is [neurologists] don't even take a look at the patient. The neurologist that is just about to arrive, he's genius in his field, really. But if I mention to him something like... hypertension, he hasn't got an idea of what to do! They order a million tests without even looking at the patients. (...) If the patient is agitated, why would you order a CT scan? It's only going to be a waste of time...

Intensivists, in contrast, were described by the nurses as 'holistic' professionals; they looked at the patient 'as a whole', monitored patients' main vital functions, and considered their overall health conditions, thus adopting an approach that nurses perceived as more appropriate and legitimate. Not only did sharing a holistic approach obscure the boundary between ICU nurses and intensivists, but it also became an 'identity marker' for many intensivists, who used it to differentiate themselves from other doctors (Reference-omitted-to-preserve-anonymity). The different forms of clinical knowledge and approaches adopted by doctors and nurses gradually 'sedimented' (Busch, 1982) to become a taken-for-granted property of specific care settings, thus affecting daily interactions between doctors and nurses in important ways.

\section{Discussion}

"Not everything is either equally negotiable or-at any given time or period of time-negotiable at all", argues Strauss. "One of the researcher's main tasks (...) is to discover just what is negotiable at any given time and place" (1978:252). With this call in mind I examined the daily interaction of doctors and nurses in three care settings, aiming to identify the features of the social and organisational context that directly influenced the construction of the medical-nursing interface. My findings contribute to the literature in three main ways.

First, although available analyses of the doctor-nurse interactions have been conducted in a variety of settings, studies that systematically compare these settings are remarkably scant. The current study sought to fill this void. It compared three care settings (a neurology ward, a neurosurgery ward, and an ICU) showing that the medical-nursing boundary can be enacted in strikingly different ways, even inside the same hospital and within the same macro-level regulatory framework. By describing in rich detail three different interactional patterns - separating, replacing, and intersecting - the study adds further nuance to the boundary-blurring/boundary-reinforcing dichotomy (Carmel, 2006; Allen, 1997) and shed new light on the flexible and contextual nature of the medical-nursing boundary (Hughes, 1988; Strauss, 1963).

Second, the study offers theoretical explanation of why the medical-nursing boundary varies across care settings and in response to which contextual factors. Although previous studies identified some reasons for these variations, these did not fully explain my findings. Allen (1997) suggested that the fragmented presence of doctors makes it difficult for doctors to sustain a rigid division of labour, thus facilitating de facto boundary blurring (Allen, 1997). Although this was consistent with my observations in the neurology and neurosurgical ward, it did not explain why boundary blurring also occurred in the ICU, where doctors were constantly present. Similarly, Carmel's (2006) conclusion that some specific features of the ICU (i.e. the contrast between the ICU and the general wards, an informal working environment, and close working interactions) may obscure the medical-nursing boundary resonated with my findings, but did not explain why boundary blurring also occurred in hospital wards that had very different features, such as the neurosurgery ward. In-depth 
examination of the collected data through a constant comparative method allowed me to identify three additional features of the 'negotiation context' that influenced the course of doctor-nurse workplace interactions. These related to the level of acuity of the ward, patients' state of awareness, and the type of clinical approach adopted by nurses and doctors.

The high level of acuity of some care settings required nurses' timely intervention and amplified the opportunities for boundary blurring (a finding that echoes Allen [1997] and Hughes [1988]). Patients' low state of awareness contributed towards making nursing and medical roles more similar in practice, thus blurring the caring-curing divide. Finally, doctors and nurses' reliance on a similar clinical approach ('holism') aligned their sense-making and, over time, became a structural property that oriented subsequent interactions. Overall, these findings advance the negotiated order perspective by disentangling the role of the 'negotiation context' and offering a framework for considering the structural properties that shape local boundary negotiations.

It is important to consider that, when taken individually, the identified properties of the negotiation context may not be capable of fully explaining boundary blurring or reinforcing. For example, my observation that the adoption of a holistic clinical approach facilitates boundary blurring may appear inconsistent with the fact that in the surgical ward boundary blurring was commonplace, despite neurosurgeons being perceived as a highly specialised medical group. In this case, other elements may have played a stronger role in obscuring the medical-nursing boundary, such as the low state of awareness of patients, the fragmented presence of the surgeons, and the acuity of the surgical ward. Similarly, whereas my findings confirm that the fragmented presence of doctors in the ward increases de facto boundary blurring (Allen, 1997), the constant presence of doctors may give rise to a range of boundary dynamics - as demonstrated by the differences between the ICU and the neurology ward. The properties of the negotiation context are thus to be conceived as part of a dynamic system rather than as a list of discrete and inflexible structures. They may interact with each other as well as other macro-level factors in meaningful ways.

Third, and finally, this study opens the way towards a more nuanced conceptualisation of power in inter-professional relationships. Negotiated order theorists move away from the strictly competitive interpretation of power proposed by the medical dominance model (Freidson, 1970) to conceptualise power as situational and contingent, varying according to the specific events at hand and negotiable despite formal organisational hierarchies (Fine, 1984). Consistent with this approach, Carmel (2006) has suggested that in the peculiar setting of the ICU, doctors and nurses may develop strategies to increase the influence of both groups in the wider network of healthcare practitioners, rather than competing with each other. The professional boundary between medicine and nursing may thus be blurred to reinforce the organisational boundary between ICU and the rest of the hospital. Similarly, Nugus et al. (2010) elaborated a distinction between 'competitive power' and 'collaborative power' to explain variations in the inter-professional negotiations they observed in different care settings. Both these studies seem to associate a collaborative use of power with a higher degree of boundary blurring, and competitive power with boundary reinforcing.

Of the three interactional patterns described by the current study, two (separating and intersecting) confirm this association. The separating pattern seems to be characterised by 'competitive power', namely a zero-sum distribution of power in which the decisions regarding patients' care are controlled by one professional group and professional boundaries are reinforced. The intersecting 
pattern resembles 'collaborative power', whereby each profession is acknowledged as having distinctive competencies, but patient care is carried out thanks to a certain amount of role interchangeability and boundary blurring. The replacing pattern presents a different scenario: the blurred medical-nursing boundary that characterised neurosurgery did not lead to a more collaborative distribution of power between doctors and nurses. Surgical nurses appeared to exercise power in ways that were not openly acknowledged by doctors but were nevertheless encouraged by their routine clinical practices. Despite occasional complaints about this informal, unrecognised job, the replacing pattern was usually maintained by both nurses and doctors, rather than openly negotiated. My findings suggest that surgical nurses' efforts to preserve their professional identities as 'hands-on workers' and 'life savers' played a substantial role in maintaining the replacing pattern. This may indicate that even ambiguous power distributions can sustain relatively stable boundary relations providing they are consistent with the identities of the professions involved. Thus, whilst confirming the significance of the collaborative-competitive model proposed by Nugus et al. (2010), the current study sheds light on a previously unexplored link between power, professional boundaries, and professional identities. This opens interesting avenues for developing our understanding of power in the negotiated order of health services.

The study presents limitations related to the methods and sample adopted. Although the data collection activities included several hours of observations of, and conversations with, 42 health providers, these were all within one hospital in Italy and in one specific clinical area. Future research should be aimed at exploring how the identified features of the 'negotiated context' operate in different clinical, organisational, and geographical settings. Other limitations may derive from the study theoretical framework. Theories provide 'sensitising concepts' that orient our way to approach empirical instances; they shed light on some aspects of the social world while overshadowing others. This study was grounded on Strauss' assertion of the 'primacy of work' in shaping the division of labour that forms around it, i.e. the idea that it is daily work practices themselves that shape division of labour (Strauss et al., 1997). Therefore, the concept of organisational culture, although not incompatible with my reflections, was not directly applied to explain variations in the medicalnursing boundary. Similarly, the impact of professionals' personality and individual differences in the regulation of the medical-nursing has been arguably underplayed.

To balance these constraints, substantial efforts were made to enhance the rigour and the theoretical relevance of my analysis. I constantly compared conversational data with observational ones, which was useful for confirming participants' accounts and probing the meaning of the observed events. Whenever feasible, I discussed my interpretations with participants to enhance my corpus of data with their 'insider accounts' (Hammersley and Atkinson 1995:124). By constantly linking my findings with those of existing studies and with a number of theoretical concepts, I sought to develop higher order interpretations that could be applied beyond their context of generation.

\section{Conclusions}

Barley and Kunda have suggested that comparative studies are essential for advancing our theories of work because they allow researchers to "articulate relations between work practices, situational contingencies, and organisational patterns" (2001:85). This study compared the construction of the medical-nursing boundary in three different care settings to disentangle the role of the 'negotiation context' (Strauss, 1978), i.e. the structural properties that directly influence interactions between 
nurses and doctors. It revealed that patients may, although unwillingly, provide or subtract opportunities for nurses to engage with a purely caring role, thus reinforcing (or blurring) the medical-nursing boundary. The type of clinical approach adopted by nurses and doctors may align (or misalign) their meaning-making processes, thus becoming, over time, a structural property that shapes subsequent interactions. Finally, as previously noted by Allen (1997) and Hughes (1988), the level of acuity of the ward may shape nurses and doctors' work in ways that encourage (or make it virtually impossible for) nurses to act strictly within their formal jurisdictions. Though further research is needed to examine how these features of the negotiation context may operate in different clinical and geographic contexts, the study advances the theory of negotiated order by offering a framework for considering the structural differences that shape local inter-professional negotiations. By describing three different doctor-nurse interactional patterns - separating, replacing, and intersecting - this study adds further nuance to the boundary-blurring/boundaryreinforcing dichotomy and sheds new light on the flexible and contextual nature of the medicalnursing interface. 


\section{References}

Abbott, A. (1988). The System of Professions: An Essay on the Division of Expert Labor. University Of Chicago Press.

Allen, D. (1997).The nursing-medical boundary: a negotiated order? Sociology of Health \& Illness, 19(4), 498-520.

Allen, D. (2014). Re-conceptualising holism in the contemporary nursing mandate: From individual to organisational relationships. Social Science \& Medicine, 119, 131-138.

Barley, S.R., \& Kunda, G. (2001). Bringing work back in. Organization science, 12(1), 76-95.

Barazzetti, G., Radaelli, S., \& Sala, R. (2005). Autonomy, responsibility and the Italian code of deontology for nurses. Nursing Ethics, 14(1), 1-29.

Baumann, A.O., Deber, R.B., Silverman, B.E., \& Mallette, C.M. (1998). Who cares? Who cures? The ongoing debate in the provision of health care. Journal of advanced nursing, 28(5), 1040-1045.

Beardshaw, V., \& Robinson, R. (1990). New for Old? Prospects for Nursing in the 1990s. London: King's Fund Institute.

Busch, L. (1982). History, structure, and negotiation in agricultural research. Urban Life, 11(3),368384.

Carmel, S. (2006). Boundaries obscured and boundaries reinforced: incorporation as a strategy of occupational enhancement for intensive care. Sociology of Health \& Illness, 28(2),154-77.

Chambliss, D.F. (1996). Beyond caring: Hospitals, nurses, and the social organization of ethics. University of Chicago Press.

Charmaz, K. (2014). Constructing grounded theory. London: Sage.

Cipolla C. \& Sena,B. (2014). "Introduzione", in Cipolla and Rocco "Dieci anni dopo: un'altra infermieristica”, Milano: FrancoAngeli, 7-21.

Dingwall, R., \& Allen, D. (2001). The implications of healthcare reforms for the profession of nursing. Nursing Inquiry, 8(2), 64-74.

Fine, G.A. (1984). Negotiated orders and organizational cultures. Annual Review of Sociology, 10, 239-262.

Foley, L. \& Faircloth, C.A. (2003). Medicine as discursive resource: legitimation in the work narratives of midwives. Sociology of Health \& Illness, 25(2), 165-184.

Freidson, E. (1970). Professional Dominance. New York: Atherton Press Inc.

Gobo, G. (2008). Doing ethnography. London: Sage.

Goopy, S.E. (2005). Taking account of local culture: Limits to the development of a professional ethos. Nursing Inquiry, 12(2), 144-154. 
Hammersley, M. \& Atkinson, P. (2007). Ethnography: Principles in practice. Abingdon: Routledge.

Hindmarsh, J., \& Pilnick, A. (2002). The tacit order of teamwork: collaboration and embodied conduct in anesthesia. The Sociological Quarterly, 43(2),139-164.

Hochschild, A.R. (2003). The managed heart: Commercialization of human feeling. University of California Press.

Hughes, E.C. (1984). The Sociological Eye: Selected Papers. New Brunswick: Transaction Press.

Hughes, D. (1988). When nurse knows best: some aspects of nurse-doctor interaction in a casualty department. Sociology of Health \& Illness, 10, 1-2.

Kangasniemi, M., Stievano, A., \& Pietilä, A.M. (2013). Nurses' perceptions of their professional rights. Nursing Ethics, 20(4), 459-69.

Kellogg, K.C. (2014). Brokerage professions and implementing reform in an age of experts. American Sociological Review, 79(5), 912-941

Nancarrow, S.A. and Borthwick, A.M. (2005). Dynamic professional boundaries in the healthcare workforce. Sociology of Health \& Illness, 27(7), 897-919.

Nyström, M. (2002). Inadequate Nursing Care in an Emergency Care Unit in Sweden Lack of a Holistic Perspective. Journal of Holistic Nursing, 20(4), 403-417.

Nugus, P., Greenfield, D., Travaglia, J., Westbrook, J., \& Braithwaite, J. (2010). How and where clinicians exercise power: Interprofessional relations in health care. Social Science \& Medicine, 71(5), 898-909.

Sansoni J. (1995). The mirror that attracts but does not shine: The long journey to recognition of nursing as a discipline. International Nurse,9:1

Salhani, D., \& Coulter, I. (2009). The politics of interprofessional working and the struggle for professional autonomy in nursing. Social Science \& Medicine, 68(7),1221-1228.

Strauss, A.L. (1978). Negotiations. Varieties, contexts, processes, and social order. San Francisco:Jossey-Bass.

Strauss, A.L, Fagerhaugh, S., Suczet, B. and Wiener, C. (1997) The Social Organization of Medical Work. New Brunswick:Transaction Press.

Tjora, A.H. (2000). The technological mediation of the nursing-medical boundary. Sociology of Health \& Illness, 22(6), 721-741.

Tousijn, W. (2002). Medical dominance in Italy: a partial decline. Social Science \& Medicine, $55(5), 733-741$.

World Health Organization (2007). Task shifting: rational redistribution of tasks among health workforce teams. www.who.int/iris/handle/10665/43821 
Yamaguchi, S. (2004). Nursing culture of an operating theater in Italy. Nursing \& health sciences, 6(4),261-269. 


\begin{tabular}{|l|l|l|l|l|}
\hline Context & Neurosurgery & Neurology & Intensive Care Unit & Total \\
\hline Hours & 68 & 52 & 105 & 225 \\
\hline
\end{tabular}

Box 1: Nursing professionalisation in Italy

In Italy the pace of nursing professionalisation has been more gradual than in other countries, but it has still been associated with significant changes in the policy and legal spheres (Yamaguchi, 2004; Cipolla and Sena, 2014). In 1991 national legislation mandated a university diploma in Nursing Science. In 1994 the Law 739/94 (known as the Professional Profile Law) formally acknowledged nursing as an autonomous profession with its own function, specific responsibilities, area of knowledge, and expertise. The Professional Profile Law had a high symbolic value for Italian nursing which, for the first time, was described as an "intellectual, competent, and autonomous profession", rather than an auxiliary healthcare occupation. A further step towards nursing autonomy was taken in 1999, when Law 42/99 abolished the Mansionario Infermieristico (literally, "nursing job description"). The Mansionario included a list of highly formalised instructions that strictly regulated nurses' daily activities, thus limiting their autonomy in daily practice. The nursing Code of Ethics was approved in 1999 (updated in 2009), through which nurses reached the status of a fully self-regulating healthcare profession (Barazzetti et al., 2005). Nurses' extended role is currently being debated in Italy, but not yet implemented.

Despite these radical shifts, anecdotal as well as scientific evidence suggests that variations in the health policies and legislations have not necessarily been reflected in workplace changes (Tousijn, 2000; Goopy, 2005; Barazzetti et al., 2005). A recent national survey showed that, despite new legislations, most nurses still complained that their legal rights are ignored by management staff because of nurses' low status within health organisations (Kangasniemi et al, 2013; Cipolla and Sena, 2014). Some have attributed the perpetuation of nursing subordination to the fact that nurses' higher education is still broadly regulated by the medical profession, with very few nurses employed as researchers or university professors (Sansoni, 1995). Others (e.g. Yamaguchi, 2004; Goopy, 2005) suggest that the limited capacity of the Italian nurses' registering body to standardise nursing practice is responsible for drawing nurses into unofficial channels of control, often regulated by doctors. The described mismatch between formal and legal structures and the daily experiences of Italian nurses, as well as the relative lack of standardisation of nursing practice, suggest that in Italy variations across care settings in how nursing jurisdiction is accomplished may be particularly pronounced, thus giving sharp profile to the salient properties of the 'negotiation context' (Strauss, 1978). 